ARTICLE

Received 29 Apr 2014 | Accepted 23 Oct 2014 | Published 12 Dec $2014 \quad$ DOl: 10.1038/ncomms6656

\title{
Competition between spontaneous symmetry breaking and single-particle gaps in trilayer graphene
}

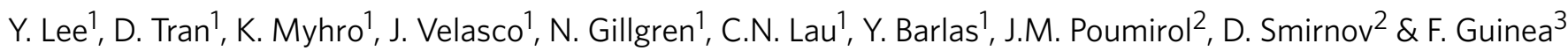

Many physical phenomena can be understood by single-particle physics; that is, treating particles as non-interacting entities. When this fails, many-body interactions lead to spontaneous symmetry breaking and phenomena such as fundamental particles' mass generation, superconductivity and magnetism. Competition between single-particle and many-body physics leads to rich phase diagrams. Here we show that rhombohedral-stacked trilayer graphene offers an exciting platform for studying such interplay, in which we observe a giant intrinsic gap $\sim 42 \mathrm{meV}$ that can be partially suppressed by an interlayer potential, a parallel magnetic field or a critical temperature $\sim 36 \mathrm{~K}$. Among the proposed correlated phases with spatial uniformity, our results are most consistent with a layer antiferromagnetic state with broken time reversal symmetry. These results reflect the interplay between externally induced and spontaneous symmetry breaking whose relative strengths are tunable by external fields, and provide insight into other low-dimensional systems.

\footnotetext{
${ }^{1}$ Department of Physics and Astronomy, University of California, Riverside, Riverside, California 91765, USA. ${ }^{2}$ National High Magnetic Field Laboratory, Tallahassee, Florida 32310, USA. ${ }^{3}$ ICMM-CSIC, E-28049 Madrid, Spain. Correspondence and requests for materials should be addressed to C.N.L. (email: jeanie.lau@ucr.edu).
} 
$\mathrm{n}$ the single-particle picture, rhombohedral trilayer graphene (r-TLG) hosts chiral charge carriers with Berry's phase $3 \pi$, and an energy-momentum dispersions $\varepsilon(k) \sim k^{3}$, thus they are gapless semiconductors (Fig. 1a inset) ${ }^{1-7}$. An energy gap can be generated via two different mechanisms, in the single-particle picture, on applying a potential difference $U_{\perp}$ between the outmost layers, the band structure adopts a gap that scales with $U_{\perp}$ (refs 1-7). Alternatively, close to the charge neutrality point (CNP), the diverging density of states leads to strong electronic interactions, and the gapless semiconductor is expected to give way to phases with spontaneous broken symmetries; in particular, gapped phases such as layer antiferromagnetic (LAF) and quantum anomalous Hall states with broken time reversal symmetry are expected to be favoured ${ }^{8-13}$. Such symmetrybroken states are in direct competition with the $U_{\perp}$-induced single-particle gap, giving rise to a rich phase diagram.

The rich phase diagram in both zero and finite magnetic field $\mathbf{B}$ in $\mathrm{r}$-TLG, lent by the competition between single-particle physics and spontaneous symmetry breaking, has not been explored to date, as most devices previously studied are singly gated and do not allow independent control of both the charge density $n$ and the single-particle term $U_{\perp}$ (refs 14-16, though a gap of $\sim 6 \mathrm{meV}$ was observed in suspended $\mathrm{r}-\mathrm{TLG}^{14}$ ).

Here we fabricate dual-gated suspended ${ }^{17-19}$ r-TLG devices ${ }^{20}$, where $U_{\perp}$ and $n$ can be independently tuned. Device mobility ranges from 20,000 to $90,000 \mathrm{~cm}^{2} \mathrm{~V}^{-1} \mathrm{~s}^{-1}$. Using low temperature transport measurements, we show that, in the absence of external fields, r-TLG at the CNP is an intrinsic insulator, with an energy gap of $42 \mathrm{meV}$; the critical temperature for transition into this insulating regime is $T_{c} \sim 36 \mathrm{~K}$. This energy gap is partially suppressed by $U_{\perp}$ or a parallel magnetic field. Among the spatially uniform correlated phases that have been proposed theoretically ${ }^{8-13}$, our experimental results are most consistent with the presence of a layer antiferromagnet in chargeneutral r-TLG, which is expected to transition into a layer polarized state and a canted ferromagnetic state on the application of external electric and magnetic fields, respectively. a

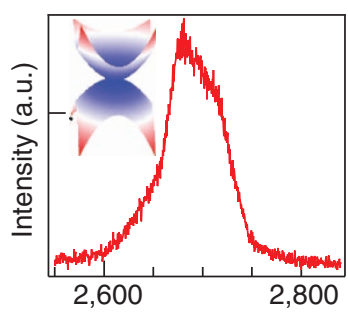

Wavenumber $\left(\mathrm{cm}^{-1}\right)$

C

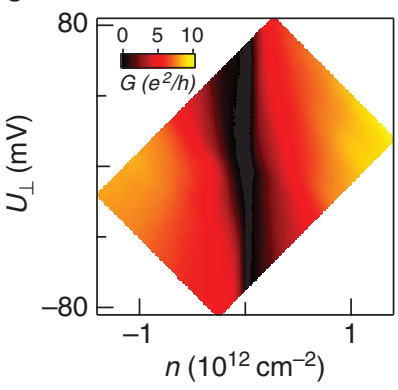

b

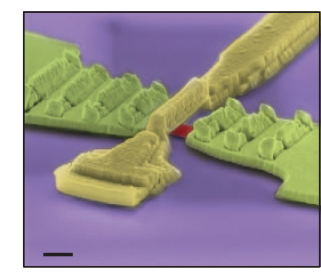

d

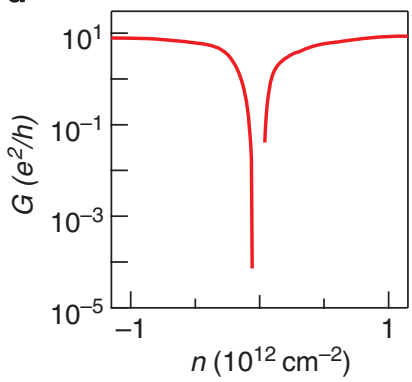

Figure 1 | Raman spectroscopy, SEM image and transport data of ABC trilayer device. (a) Raman spectroscopy of $r$-TLG sheet. Inset: energymomentum dispersion of $r-T L G$. (b) SEM image of a dual-gated suspended TLG device. Scale bar, $1 \mu \mathrm{m}$. (c) $G\left(U_{\perp}, n\right)$ in units of $e^{2} / h$. (d) $G(n)$ at $U_{\perp}=0$. Note the logarithmic scale of $G$.

\section{Results}

Gapped insulating state at the CNP. r-TLG sheets on $\mathrm{Si} / \mathrm{SiO}_{2}$ are identified by Raman spectroscopy ${ }^{21,22}$ (Fig. 1a), suspended dual-gated devices (Fig. 1b) with mobility as high as $90,000 \mathrm{~cm}^{2} \mathrm{~V}^{-1} \mathrm{~s}^{-1}$ are measured in He-3 refrigerators. All measurements are taken at $T=260 \mathrm{mK}$, unless otherwise specified.

At low temperatures and $\mathbf{B}=0, r$-TLG devices become insulating in the vicinity of the CNP. In Fig. 1c, the two-terminal differential conductance $G=d I / d V$ from device 1 is plotted as $n$ and $U_{\perp}$. At $U_{\perp}=0$, as $n$ approaches the CNP, $G(n)$ decreases by more than five orders of magnitude to $<10^{-4} e^{2} / h$, where $e$ is electron charge and $h$ is Planck's constant (Fig. 1d). This insulating state at the CNP is extremely robust, as it persists for the entire gate ranges up to the highest applied $U_{\perp}(\sim 65 \mathrm{mV})$.

Transport spectroscopy ${ }^{17,19}$ at $n=U_{\perp}=0$ reveals intriguing features: at small source-drain bias $V$, the device stays insulating, but as $V$ increases $\pm 42 \mathrm{mV}, G$ rises by more than six orders of magnitude to extremely sharp peaks, then decreases to $\sim 15 e^{2} / h$ for larger $V$ (Fig. 2a). Such a $G(V)$ curve strongly resembles the density of state of a gapped phase, suggesting the presence of an energy gap $\Delta \sim 42 \mathrm{meV}$ at $n=U_{\perp}=\mathbf{B}=0$. With increasing charge density $n$, the gap diminishes and eventually disappears entirely (Fig. 2b). At $n=3 \times 10^{11} \mathrm{~cm}^{-2}, G(V)$ is flat, indicating that $\mathrm{r}-\mathrm{TLG}$ becomes gapless at high density.

The gapped, insulating state near the CNP in the absence of external fields is unexpected from tight-binding calculations, but instead suggests a phase arising from electronic interactions with spontaneous broken symmetries ${ }^{23}$. The magnitude of the gap, $\sim 42 \mathrm{meV}$, is exceedingly large for an interaction-induced state. It is more than an order of magnitude larger than that found in $\mathrm{BLG}^{17,24}$, reflecting the divergent nature of the density of states and strong electronic interactions in r-TLG at the CNP. It also a factor of seven larger than that previously observed in singly gated devices ${ }^{14}$, likely due to improved device geometry and quality.

Temperature dependence of the gapped state. To further establish the magnitude of the gap, we examine temperature dependence of $G(V)$ at $n=U_{\perp}=0$ (Fig. 2c). Figure 2d plots minimum conductance $G_{\min }=G(V=0)$ as a function of $T$. At high temperatures $T>40 \mathrm{~K}$, the device is conductive, $G_{\min } \sim 15 e^{2} / h$ with a small linear $T$-dependence. The $G(V)$ curves are approximately constant, similar to that of a conventional resistor. However, when $T<\sim 40 \mathrm{~K}, G_{\min }$ drops precipitously and becomes insulating for $T<30 \mathrm{~K}$, and $G(V)$ curves develop prominent peaks at finite $V$. In the transition region $30<T<40 \mathrm{~K}$, the $G_{\min }(T)$ curve is well-described by the thermal activation equation, $G_{\min }=G_{0} e^{-\Delta / 2 k_{B} T} \quad$ (Fig. 2e), where $k_{\mathrm{B}}$ is the Boltzmann's constant and $\Delta \sim 43 \mathrm{meV}$ is obtained as a fitting parameter. This is in excellent agreement with the value of $\Delta$ obtained from $G(V)$ curves at $T=300 \mathrm{mK}$, thus confirming the presence of an insulating state with $\sim 42 \pm 1 \mathrm{meV}$ gap.

Using $G(V)$ curves, we can also directly measure the evolution of $\Delta$ (taken as half of the peak-to-peak separation in $V$ ) as a function of $T$. As shown in Fig. 2f, $\Delta$ is almost constant for $T<10 \mathrm{~K}$, but drops precipitously for $T>30 \mathrm{~K}$. This behaviour is a characteristic of order parameters during phase transitions in mean field theories. Thus, we fit $\Delta(T)$ to the function ${ }^{25}$

$$
\Delta(T)=\Delta(0)\left[A\left(1-\frac{T}{T_{c}}\right)+B\left(1-\frac{T}{T}\right)^{2}\right]^{1 / 2}
$$

where $T_{c}$ is the critical temperature. Equation (1) reduces to the usual mean -field functional form $\sqrt{1-\frac{T}{T_{c}}}$ for $T / T_{\mathrm{c}}$ sufficiently 

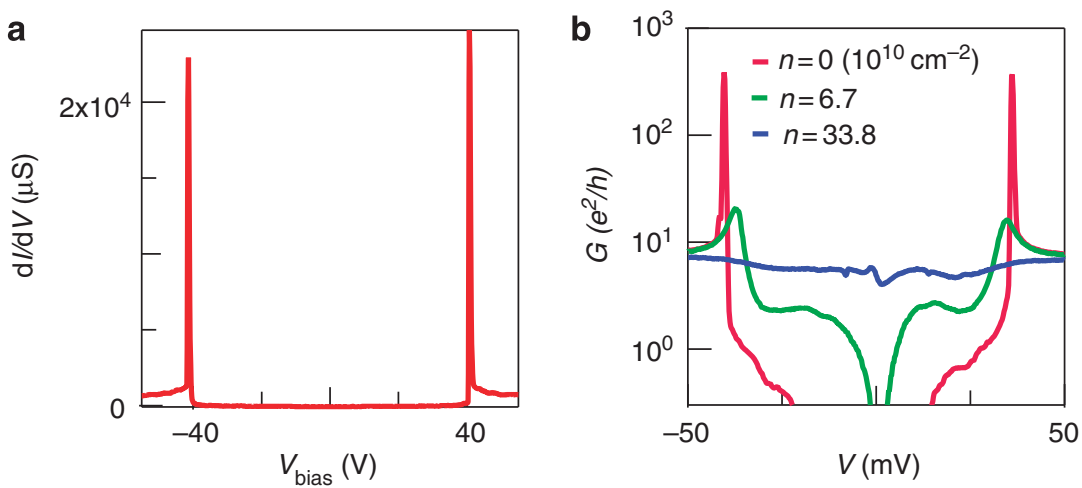

C

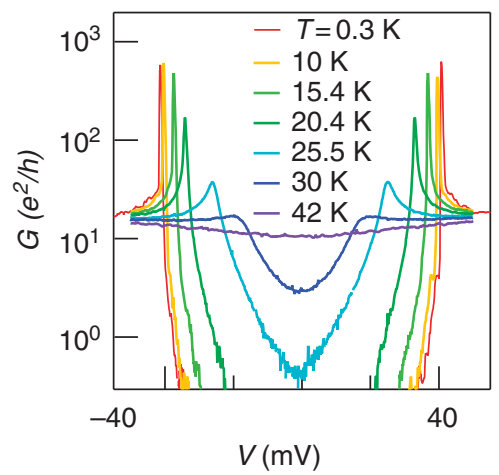

d

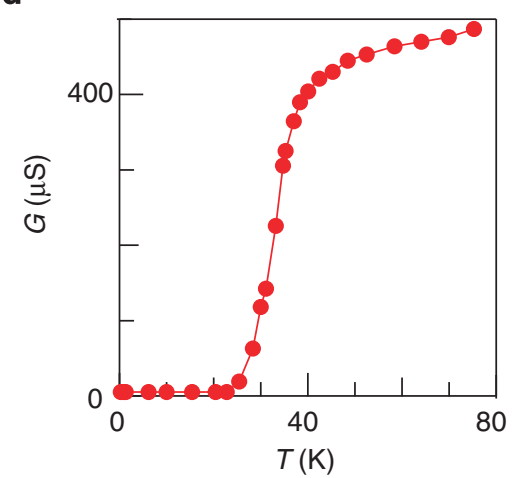

e

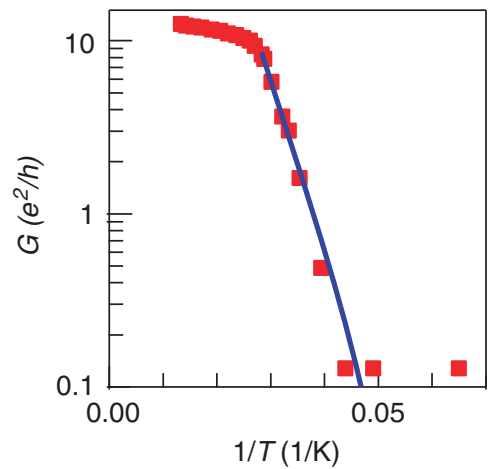

f

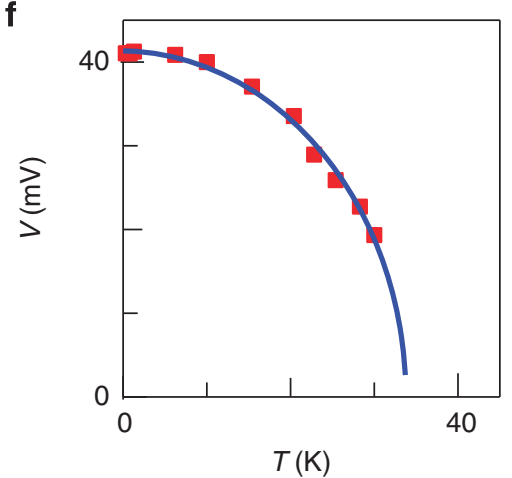

Figure 2 | Transport data at $\boldsymbol{B}_{\perp}=\mathbf{O}$ (a,b: Device 1; c-f: Device 2). (a) $G(V)$ at $U_{\perp}=n=0$. (b) $G(V)$ at $U_{\perp}=0$ and different $n$. (c) $G_{\min }(V)$ at $U_{\perp}=n=0$ and different temperatures. (d) $G_{\min }$ at $V=0$ versus $T$. (e) $G_{\min }$ versus $1 / T$ in Arrhenius scale. The blue line is a fit to the equation $G_{\min }=G_{0} e^{-\Delta / 2 k_{B} T}$ for $30 \mathrm{~K}<T<40 \mathrm{~K}$. (f) Measured $\Delta$ as a function of $T$. The solid line is a fit to equation (1).

close to 1 , and the second term $\left(1-\frac{T}{T_{c}}\right)^{2}$ is inserted to capture the vanishingly small dependence on $T$ as $T \rightarrow 0$. Excellent agreement with data is obtained, yielding $A=2.0, B=-1.0$ and $T_{\mathrm{c}}=34 \mathrm{~K}$. The energy scale of the gap, $\Delta(0) / k_{\mathrm{B}}=500 \mathrm{~K}$, which is much larger than that associated with the critical temperature, signifies that this insulating state observed at the CNP is a correlated phase.

$E$ and $B_{\|}$field dependence of the gapped state. To elucidate the nature of this correlated phase, we examine how it is modified in the presence of an external field that selectively breaks one of the degeneracies. For instance, application of $U_{\perp}$ breaks the inversion (which-layer) symmetry, and, in the single-particle picture, gives rise to a proportionally scaled energy gap. Figure 3a displays $G$ as a function of $V$ and $U_{\perp}$ at $n=0$. As $U_{\perp}$ is the externally imposed potential bias, it will be heavily screened due to r-TLG's large density of states near the CNP $26-28$. Thus, we expect the screened interlayer potential bias $U_{\perp}^{s} \ll U_{\perp}$. Using a simplified two-band
Hamiltonian for $\mathrm{r}$-TLG and assuming that the dielectric constant of $\mathrm{r}$-TLG is 1 , we self-consistently calculate $U_{\perp}^{s}$ for given values of $n$ and $U_{\perp}$ (see Supplementary Note 1 for detail):

$$
U_{\perp}^{s}=U_{\perp}+\frac{d e^{2}}{2 \varepsilon_{o}}\left(\frac{1}{\pi}\right)\left(\frac{\gamma_{1}}{\hbar v}\right)^{2}\left(\frac{U_{\perp}^{s}}{2 \gamma_{1}}\right)^{2 / 3}\left[\int_{0}^{x_{F}} \frac{d x}{\sqrt{1+x^{3}}}-2.8\right]
$$

where $x_{F}=\left(\frac{4}{\left(U_{\perp}^{s}\right)^{2} \gamma_{1}^{4}}\right)^{\frac{1}{3}} v^{2} \pi \hbar^{2} n, \quad \gamma_{1}$ is the interlayer hopping parameter, $v$ the Fermi velocity of charges in monolayer graphene, $d=0.67 \mathrm{~nm}$ is the spacing between outmost layers (for derivation of equation (2), see Supplementary Note 1). The screening-corrected data $G\left(V, U_{\perp}^{s}\right)$ are shown in Fig. 3b.The sharp peaks in $G(V)$, that is, the gap edges, appear as red curves that separate the insulating (dark blue) and conductive (light blue) regions in Fig. 3a,b. $\Delta$ decreases symmetrically and linearly with applied $U_{\perp}^{s}$ of either polarity, to $\sim 30 \mathrm{meV}$ at $\left|U_{\perp}\right|=50 \mathrm{mV}$ or $\left|U_{\perp}^{s}\right|=3 \mathrm{mV}$ (Fig. $3 \mathrm{~b}$ ), though not yet completely closed at the 
a

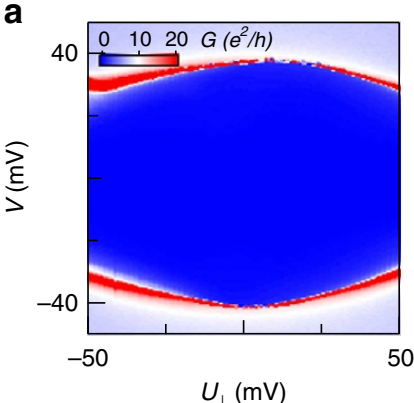

C

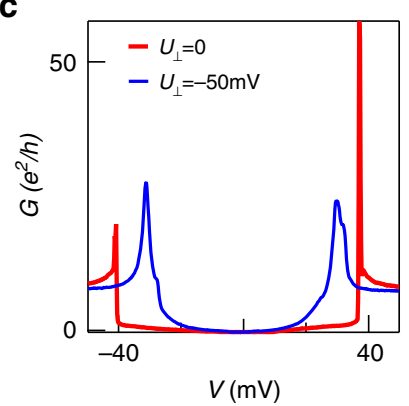

b

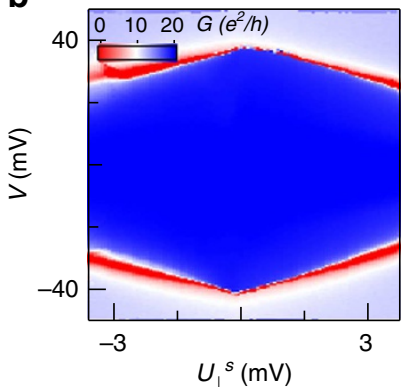

d

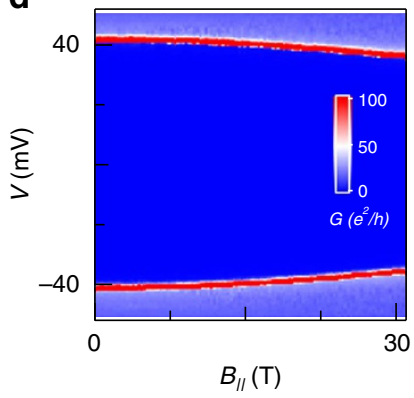

Figure 3 | Transport data at $\boldsymbol{n}=\mathbf{0}$ and finite $\boldsymbol{U}_{\perp}$ and $\boldsymbol{B}_{\| \cdot}(\mathbf{a}, \mathbf{b}) G\left(V, U_{\perp}\right)$ and $G\left(V, U_{\perp}^{s}\right)$ in units of $e^{2} / h$ from Device 1. (c) Line traces $G(V)$ at $U_{\perp}=0$ and $U_{\perp}=-50 \mathrm{mV}$. (d) $G\left(V, B_{\|}\right)$in units of $e^{2} / h$ from Device 2 .

largest applied $\left|U_{\perp}\right|$. (In other devices with lower mobility, we also observe that the device conductance increases from $<0.1 e^{2} / h$ with increasing $\left|U_{\perp}\right|$, exceeding $1.5 e^{2} / h$ at $\left|U_{\perp}\right|=85 \mathrm{mV}$, as shown in Supplementary Fig. 2).

In contrast, an in-plane magnetic field $B_{\|}$couples to the spin but not orbital degrees of freedom and raises the Zeeman energy. Figure $3 \mathrm{~d}$ plots $G\left(V, B_{\|}\right)$for $B_{\|}=0$ to $31 \mathrm{~T}$. $\Delta$ stays almost constant for $B_{\|}<10 \mathrm{~T}$, but decreases to $35 \mathrm{mV}$ at $B_{\|}=31 \mathrm{~T}$. Thus, addition of Zeeman energy suppresses the gapped phase, suggesting that the phase has spin-ordering, but no net magnetic moment.

\section{Discussion}

To summarize our experimental findings, we observe an insulating state in r-TLG at $n=U_{\perp}=\mathbf{B}=0$, with an energy gap $\Delta(T=0) \sim 42 \mathrm{meV}$. This gap can be suppressed by increasing charge density $n$, a critical temperature $T_{\mathrm{c}} \sim 34 \mathrm{~K}$, by an interlayer potential $U_{\perp}$ of either polarity and by an in-plane magnetic field. Among the spatially uniform correlated phases in r-TLG discussed in the literature ${ }^{8-13}$, only LAF, in which the top and bottom layers have equal number of electrons with opposite spin polarization, is consistent with our experimental observations. For instance, the presence of an energy gap eliminates the mirrorbreaking, inversion breaking, interlayer current density wave or layer polarization density wave states ${ }^{12}$, and the zero conductance eliminates the superconductor, quantum spin Hall and quantum anomalous Hall states that host finite (or even infinite) conductance. Furthermore, the symmetrical suppression of the gap by $U_{\perp}$ of either polarity suggests that charges in the insulating state are layer-balanced, as the device would otherwise exhibit opposite dependence on $U_{\perp}$ of opposite polarities. This excludes all layer-polarized states, including the quantum valley Hall and layer polarization density wave states, and any single-particle state that arises from inadvertent doping of one of the surface layers.

Thus, based on the above experimental observations, we identify LAF with broken time reversal and spin rotation symmetries as the most likely candidate among the proposed ground states in r-TLG. Theoretically, an LAF ground state can be justified by the following considerations. The strong screening

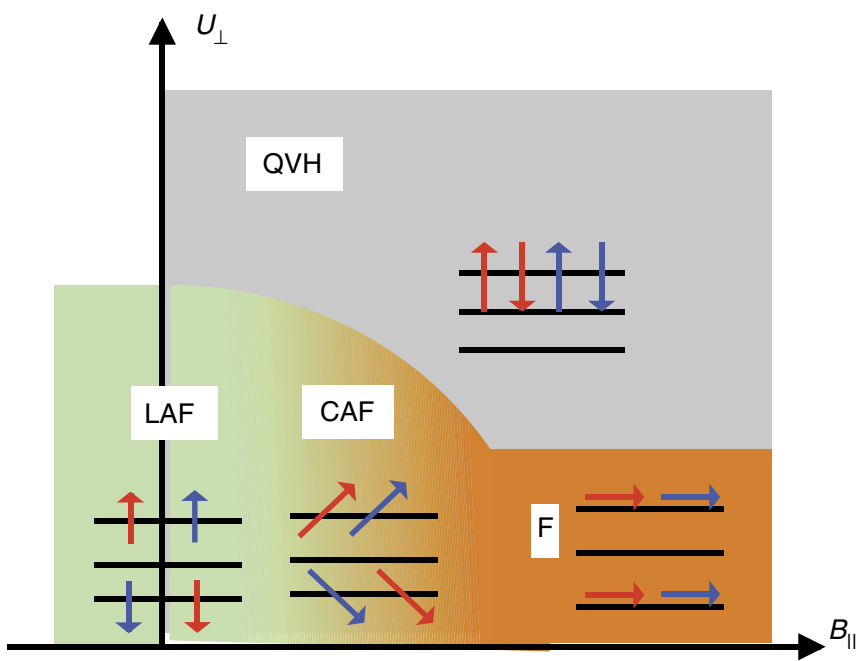

Figure 4 | Possible phase diagram and schematics of electronic configurations for $\mathbf{r}$-TLG. The blue and red arrows indicate charges from $\mathrm{K}$ and $\mathrm{K}^{\prime}$ valleys, respectively. (CAF, canted antiferromagnet; F, ferromagnet; LAF, layer antiferromagnet; QVH, quantum valley Hall).

due to the large density of states in r-TLG leads to very short range Coulomb repulsions among electrons (see Supplementary Note 3 ). For such local interactions, the most likely symmetrybroken states are the LAF and nematic phases, and the gapless and conductive nematic phase is incompatible with our observation of a gapped insulator, leaving LAF as the only viable alternative. Moreover, mean field arguments that generally favour the LAF phase should be more robust in r-TLG than that in bilayer graphene, due to the divergent density of states and stronger electrons near the CNP that suppresses fluctuations. Finally, recent works ${ }^{29,30}$ report formation of magnetic moments in graphene that results from interactions among the $\pi$-electrons of graphene in the presence of hydrogen and fluorine atoms and vacancies, thus it is not unreasonable to expect emergent magnetism arising from strong electronic interactions in r-TLG.

Within the mean-field framework, a simple estimate of the LAF gap yields (see Supplementary Note 4)

$$
\Delta \approx 2 \times\left(\frac{c}{\pi \sqrt{3}}\right)^{3} \frac{\gamma_{1}^{4} U^{3}}{\gamma_{0}^{6}}
$$

where $\gamma_{0} \approx 2.7 \mathrm{eV}, \gamma_{1} \approx 0.4 \mathrm{eV}$ are tight binding parameters, $c \approx 2.8$, and $U$ is the Hubbard onsite interaction. Using these parameters, and substituting the experimentally obtained value $\Delta=42 \mathrm{meV}$, we obtain $U \sim 13 \mathrm{eV}$, not too different from theoretically predicated values of $5-10 \mathrm{eV}$ (refs 31-33). Alternatively, the gap can be further enhanced by exchange processes associated with the long-range part of the interaction ${ }^{31}$.

A possible phase diagram for charge neutral $r$-TLG that is consistent with our experimental results, together with schematics for electron configurations, is summarized in Fig. 4. In the absence of external fields, a charge neutral r-TLG is an LAF with broken time reversal and spin rotation symmetries. Increasing $U_{\perp}$ of either polarity pushes electrons to one of the surface layers and suppresses the gap. For sufficiently large $\left|U_{\perp}\right|$, all charges reside in either the top or bottom layer, giving rise to a quantum valley Hall (QVH) insulator with broken inversion symmetry. We note that the different broken symmetries of the QVH and LAF states preclude a continuous phase transition between them ${ }^{5,34,35}$. In contrast, as $B_{\|}$increases from 0 , the competition between the Zeeman and the exchange energies tilts the electron spins, and r-TLG crosses over to the CAF phase. For very large $B_{\|}$, we expect that the electrons eventually form a ferromagnet $(F)$; in the 
quantum Hall regime, this ferromagnet is analogous to a quantum spin Hall state, with counter-propagating edge states and conductance $\sim 6 e^{2} / h$ (ref. 36).

\section{Methods}

Sample preparation. Trilayer graphene sheets are mechanically exfoliated onto $300 \mathrm{~nm} \mathrm{SiO}{ }_{2}$ wafer layer grown over degenerately doped $\mathrm{Si}$. $\mathrm{ABC}$ or rhomborohedral stacked trilayer ( $r$-TLG) sheets are selected by colour contrast in optical microscope ${ }^{37}$ and Raman spectroscopy ${ }^{21,22}$, coupled to electrodes and top gates using multi-level lithography ${ }^{38,39}$, and etched in $\mathrm{HF}$ to partially remove the $\mathrm{SiO}_{2}$ layer. All devices have source-drain separation $\sim 1.1 \mu \mathrm{m}$ and width $\sim 1.1-1.4 \mu \mathrm{m}$.

Characterization of devices. Most as-fabricated devices have low mobility, and current annealing is performed at $4 \mathrm{~K}$ to improve sample quality ${ }^{40,41}$. Typically the optimal annealing results are achieved when current starts to saturate, at $\sim 0.3 \sim 0.4 \mathrm{~mA} \mathrm{\mu m}^{-1}$ layer ${ }^{-1}$. After annealing, device mobility is as high as $90,000 \mathrm{~cm}^{2} \mathrm{~V}^{-1} \mathrm{~s}^{-1}$. Supplementary Figure 1 displays the conductance $G$ of a typical r-TLG device as a function of back gate voltage $V_{\mathrm{bg}}$ before and after annealing. The field-effect mobility of this device after annealing is

$\sim 50,000 \mathrm{~cm}^{2} \mathrm{~V}^{-1} \mathrm{~s}^{-1}$, with a minimum conductivity $\sim 0$. The devices are measured in helium-3 refrigerators using standard lock-in techniques.

Calculation of charge density and interlayer potential. For a given device, the induced charge density $n$ and interlayer potential $U_{\perp}$ are calculated from the back gate and top gate voltages, $n=\left(C_{\mathrm{bg}} V_{\mathrm{bg}}+C_{\mathrm{tg}} V_{\mathrm{tg}}\right) / e$ and $U_{\perp}=\left(C_{\mathrm{bg}} V_{\mathrm{bg}}-C_{\mathrm{tg}} V_{\mathrm{tg}}\right)$ $d / 2 \varepsilon_{0}$. Here $e$ is electron charge, $\varepsilon_{0}$ the permittivity of vacuum, $d=0.67 \mathrm{~nm}$ is the distance between the top and bottom layers, $V_{\mathrm{bg}}$ and $V_{\mathrm{tg}}$ are the voltage applied to back gate and top gate, respectively, and $C_{\mathrm{bg}}\left(C_{\mathrm{tg}}\right)$ is the capacitance per unit area between graphene and back (top) gate. Capacitances are estimated from geometric considerations, as well as from standard Landau level fan diagrams. For typical devices, $C_{\mathrm{bg}} / e$ ranges from $3 \times 10^{10}$ to $5 \times 10^{10} \mathrm{~cm}^{-2}$, and $C_{\mathrm{tg}} / e$ from $1 \times 10^{10}$ to $7 \times 10^{10} \mathrm{~cm}^{-2}$.

\section{References}

1. Koshino, M. \& McCann, E. Trigonal warping and Berry's phase N pi in ABCstacked multilayer graphene. Phys. Rev. B 80, 165409 (2009).

2. Guinea, F., Castro Neto, A. H. \& Peres, N. M. R. Electronic states and Landau levels in graphene stacks. Phys. Rev. B 73, 245426 (2006).

3. Avetisyan, A. A., Partoens, B. \& Peeters, F. M. Stacking order dependent electric field tuning of the band gap in graphene multilayers. Phys. Rev. B 81, 115432 (2010)

4. Aoki, M. \& Amawashi, H. Dependence of band structures on stacking and field in layered graphene. Solid State Commun. 142, 123-127 (2007).

5. Zhang, F., Sahu, B., Min, H. K. \& MacDonald, A. H. Band structure of ABC-stacked graphene trilayers. Phys. Rev. B 82, 035409 (2010).

6. Zou, K., Zhang, F., Capp, C., MacDonald, A. H. \& Zhu, J. Transport studies of dual-gated $A B C$ and $A B A$ trilayer graphene: band gap opening and band structure tuning in very large perpendicular electric fields. Nano Lett. 13, 369-373 (2013).

7. Lui, C. H., Li, Z., Mak, K. F., Cappelluti, E. \& Heinz, T. F. Observation of an electrically tunable band gap in trilayer graphene. Nat. Phys. 7, 944-947 (2011).

8. Castro, E. V., Pilar Lopez-Sancho, M. \& Vozmediano, M. A. H. Vacancy induced zero energy modes in graphene stacks: The case of ABC trilayer. Solid State Commun. 152, 1483-1488 (2012).

9. Olsen, R., van Gelderen, R. \& Smith, C. M. Ferromagnetism in ABC-stacked trilayer graphene. Phys. Rev. B 87, 115414 (2013).

10. Liu, H., Jiang, H., Xie, X. C. \& Sun, Q.-f. Spontaneous spin-triplet exciton condensation in ABC-stacked trilayer graphene. Phys. Rev. B 86, 085441 (2012).

11. Jung, J. \& MacDonald, A. H. Gapped broken symmetry states in ABC trilayer graphene. Preprint at http://arXiv.org/abs/1208.0116 (2012).

12. Cvetkovic, V. \& Vafek, O. Topology and symmetry breaking in ABC trilayer graphene. Preprint at http://arXiv.org/abs/1210.4923 (2012).

13. Scherer, M. M., Uebelacker, S., Scherer, D. D. \& Honerkamp, C. Interacting electrons on trilayer honeycomb lattices. Phys. Rev. B 86, 155415 (2012).

14. Bao, W. et al. Stacking-dependent band gap and quantum transport in trilayer graphene. Nat. Phys. 7, 948-952 (2011).

15. Bao, W. Z. et al. Magnetoconductance oscillations and evidence for fractional quantum Hall states in suspended bilayer and trilayer graphene. Phys. Rev. Lett. 105, 246601 (2010).

16. Elferen, H. J. v. et al. Fine structure of the lowest Landau level in suspended trilayer graphene. Phys. Rev. B 88, 121302(R) (2013)

17. Velasco, J. et al. Transport spectroscopy of symmetry-broken insulating states in bilayer graphene. Nat. Nanotech. 7, 156-160 (2012).

18. Lee, Y. et al. Broken symmetry quantum Hall states in dual gated ABA trilayer grapehene. Nano Lett. 13, 1627-1631 (2013).

19. Velasco, Jr J. et al. Transport measurement of Landau level gaps in bilayer graphene with layer polarization control. Nano Lett. 14, 1324-1328 (2014).
20. Khodkov, T., Withers, F., Hudson, D. C., Craciun, M. F. \& Russo, S. Electrical transport in suspended and double gated trilayer graphene. Appl. Phys. Lett. 100, 013114 (2012).

21. Lui, C. H. et al. Imaging stacking order in few-layer graphene. Nano Lett. 11, 164-169 (2011)

22. Ferrari, A. C. et al. Raman spectrum of graphene and graphene layers. Phys. Rev. Lett. 97, 187401 (2006).

23. Jia, J., Gorbar, E. V. \& Gusynin, V. P. Gap generation in ABC-stacked multilayer graphene: Screening versus band flattening. Phys. Rev. B 88, 205428 (2013).

24. Freitag, F., Trbovic, J., Weiss, M. \& Schonenberger, C. Spontaneously gapped ground state in suspended bilayer graphene. Phys. Rev. Lett. 108, 076602 (2012).

25. Ferrell, R. A. Temperature dependence of the superconductivity energy gap. Zeitschrift fur Physik 182, 1 (1964)

26. van Gelderen, R., Olsen, R. \& Smith, C. M. Screening in multilayer graphene. Preprint at http://arXiv.org/abs/1304.5501 (2013).

27. Koshino, M. Interlayer screening effect in graphene multilayers with $A B A$ and ABC stacking. Phys. Rev. B 81, 125304 (2010).

28. Min, H., Hwang, E. H. \& Das Sarma, S. Polarizability and screening in chiral multilayer graphene. Phys. Rev. B 86, 081402 (2012).

29. Nair, R. R. et al. Spin-half paramagnetism in graphene induced by point defects. Nat. Phys. 8, 199-202 (2012).

30. Nair, R. R. et al. Dual origin of defect magnetism in graphene and its reversible switching by molecular doping. Nat. Commun. 4, 2010 (2013).

31. Wehling, T. O. et al. Strength of effective coulomb interactions in graphene and graphite. Phys. Rev. Lett. 106, 236805 (2011).

32. Verges, J. A., SanFabián, E., Chiappe, G. \& Louis, E. Fit of Pariser-Parr-Pople and Hubbard model Hamiltonians to charge and spin states of polycyclic aromatic hydrocarbons. Phys. Rev. B 81, 085120 (2010).

33. Schüler, M., Rösner, M., Wehling, T. O., Lichtenstein, A. I. \& Katsnelson, M. I Optimal Hubbard models for materials with nonlocal Coulomb interactions: graphene, silicene, and benzene. Phys. Rev. Lett. 111, 036601 (2013).

34. Jung, J., Zhang, F. \& MacDonald, A. H. Lattice theory of pseudospin ferromagnetism in bilayer graphene: competing interaction-induced quantum Hall states. Phys. Rev. B 83, 115408 (2011).

35. Zhang, F., Tilahun, D. \& MacDonald, A. H. Hund's rules for the $\mathrm{N}=0$ Landau levels of trilayer graphene. Phys. Rev. B 85, 165139 (2012).

36. Maher, P. et al. Evidence for a spin phase transition at charge neutrality in bilayer graphene. Nat. Phys. 9, 154-158 (2013).

37. Craciun, M. F. et al. Trilayer graphene is a semimetal with a gate-tunable band overlap. Nat. Nanotech. 4, 383-388 (2009).

38. Liu, G., Velasco, J., Bao, W. Z. \& Lau, C. N. Fabrication of graphene p-n-p junctions with contactless top gates. Appl. Phys. Lett. 92, 203103 (2008).

39. Velasco, J., Liu, G., Bao, W. Z. \& Lau, C. N. Electrical transport in high-quality graphene pnp junctions. New J. Phys. 11, 095008 (2009).

40. Moser, J., Barreiro, A. \& Bachtold, A. Current-induced cleaning of graphene. Appl. Phys. Lett. 91, 163513 (2007).

41. Bao, W. et al. Evidence for a spontaneous gapped state in ultraclean bilayer graphene. Proc. Natl Acad. Sci. USA 109, 10802-10805 (2012).

\section{Acknowledgements}

We thank R. Cote for discussions. Y.L., K.M. and C.N.L. are supported by DOE BES Division under grant no. ER 46940-DE-SC0010597. Part of this work was performed at NHMFL that is supported by NSF/DMR-0654118, the State of Florida, and DOE. D.T. is supported by NSF DMR/1106358. Y.B. is supported by CONSEPT center at UCR. Nanofabrication and supplies are supported in part by DMEA H94003-10-2-1003 and CONSEPT center.

\section{Author contributions}

C.N.L., Y.L. and J.V. conceived the experiments. D.T. and N.G. isolated and identified r-TLG sheets. Y.L., K.M. and J.V. fabricated samples. Y.L., K.M., J.V., J.M.P. and D.S. performed measurements. C.N.L. and Y.L. analysed data. Y.B. and P.G. performed theoretical calculations. C.N.L., Y.L., Y.B. and P.G. interpreted data and wrote the manuscript. All authors discussed the results and commented on the manuscript.

\section{Additional information}

Supplementary Information accompanies this paper at http://www.nature.com/ naturecommunications

Competing financial interests: The authors declare no competing financial interests.

Reprints and permission information is available online at http://npg.nature.com/ reprintsandpermissions/

How to cite this article: Lee, Y. et al. Competition between spontaneous symmetry breaking and single-particle gaps in trilayer graphene. Nat. Commun. 5:5656 doi: $10.1038 /$ ncomms6656 (2014) 